\title{
Some Remarks on Nonlinear Electrodynamics
}

\author{
Patricio Gaete \\ Departamento de Física and Centro Científico-Tecnológico de Valparaíso, Universidad Técnica Federico Santa María, \\ Casilla 110-V, Valparaíso, Chile \\ Correspondence should be addressed to Patricio Gaete; patricio.gaete@usm.cl
}

Received 13 October 2015; Revised 15 December 2015; Accepted 27 December 2015

Academic Editor: Elias C. Vagenas

Copyright (C) 2016 Patricio Gaete. This is an open access article distributed under the Creative Commons Attribution License, which permits unrestricted use, distribution, and reproduction in any medium, provided the original work is properly cited. The publication of this article was funded by SCOAP $^{3}$.

\begin{abstract}
By using the gauge-invariant, but path-dependent, variables formalism, we study both massive Euler-Heisenberg-like and EulerHeisenberg-like electrodynamics in the approximation of the strong-field limit. It is shown that massive Euler-Heisenberg-type electrodynamics displays the vacuum birefringence phenomenon. Subsequently, we calculate the lowest-order modifications to the interaction energy for both classes of electrodynamics. As a result, for the case of massive Euler-Heisenbeg-like electrodynamics (Wichmann-Kroll), unexpected features are found. We obtain a new long-range $\left(1 / r^{3}\right.$-type) correction, apart from a long-range $\left(1 / r^{5}\right.$-type $)$ correction to the Coulomb potential. Furthermore, Euler-Heisenberg-like electrodynamics in the approximation of the strong-field limit (to the leading logarithmic order) displays a long-range $\left(1 / r^{5}\right.$-type) correction to the Coulomb potential. Besides, for their noncommutative versions, the interaction energy is ultraviolet finite.
\end{abstract}

\section{Introduction}

The phenomenon of vacuum polarization in quantum electrodynamics (QED), arising from the polarization of virtual electron-positron pairs and leading to nonlinear interactions between electromagnetic fields, remains as exciting as in the early days of QED [1-5]. An example that illustrates this is the scattering of photons by photons, which despite remarkable progress has not yet been confirmed [6-10]. Along the same line, we also recall that alternative scenarios such as BornInfeld theory [11], millicharged particles [12], or axion-like particles [13-15] may have more significant contributions to photon-photon scattering physics.

Interestingly, it should be recalled here that the physical effect of vacuum polarization appears as a modification in the interaction energy between heavy charged particles. In fact, this physical effect changes both the strength and the structural form of the interaction energy. This clearly requires the addition of correction terms in the Maxwell Lagrangian to incorporate the contributions from vacuum polarization process. Two important examples of such a class of contributions are the Uehling and Serber correction and the Wichmann-Kroll correction, which can be derived from the
Euler-Heisenberg Lagrangian. Incidentally, as explained in [5], it is of interest to notice that the Euler-Heisenberg result extends the Euler-Kockel calculation (in the constant background field limit), which contains nonlinear corrections in powers of the field strengths, whereas the Uehling and Serber result contains corrections linear in the fields (but nonlinear in the space-time dependence of the background fields). We further mention that, as in the Euler-Heisenbeg case, BornInfeld (BI) electrodynamics also contains similar nonlinear corrections to Maxwell theory from a classical point of view, as is well known. Nevertheless, BI electrodynamics is distinguished, since BI-type effective actions arise in many different contexts in superstring theory [16, 17]. In addition to Born-Infeld theory, other types of nonlinear electrodynamics have been discussed in the literature [18-23].

In this perspective, we also point out that extensions of the Standard Model (SM) such as Lorentz invariance violating scenarios and fundamental length have become the focus of intense research activity [24-31]. This has its origin in the fact that the SM does not include a quantum theory of gravitation, so as to circumvent difficulties theoretical in the quantum gravity program. Within this context quantum field theories allowing noncommuting position operators have 
been studied by using a star-product (Moyal product) [3237]. In this connection, it becomes of interest, in particular, to recall that a novel way to formulate noncommutative quantum field theory has been proposed in [38-40]. The key ingredient of this development is to introduce coherent states of the quantum position operators [41], where a modified form of heat kernel asymptotic expansion which does not suffer from short distance divergences has been obtained. We also point out that an alternative derivation of the coherent state approach has been implemented through a new multiplication rule which is known as Voros starproduct [42]. Anyhow, physics turns out to be independent of the choice of the type of product [43]. It is worthy to note here that this type of noncommutativity (coherent state approach) leads to a smearing effect which is equivalent to that encountered in a class of nonlocal theory. In other words, noncommutativity is just a subclass of possible nonlocal deformation $[44,45]$. More recently, this new approach has been successfully extended to black holes physics [46], also in connection to holographic superconductors via AdS-CFT [47].

Inspired by these observations, the purpose of this paper is to extend our previous studies $[18,19]$ on nonlinear electrodynamics to the case when vacuum polarization corrections are taken into account. The preceding studies were done using the gauge-invariant but path-dependent variables formalism, where the interaction potential energy between two static charges is determined by the geometrical condition of gauge invariance. One important advantage of this approach is that it provides a physically based alternative to the usual Wilson loop approach. Accordingly, we will work out the static potential for electrodynamics which include, apart from the Maxwell Lagrangian, additional terms corresponding to the Uehling, massive Euler-Heisenberg-like, and EulerHeisenberg electrodynamics in the approximation of the strong-field limit (to the leading logarithmic order) and for their noncommutative versions. Our results show a longrange $1 / L^{5}$-type correction to the Coulomb potential for both massive Euler-Heisenberg-like and Euler-Heisenberg electrodynamics in the approximation of the strong-field limit (to the leading logarithmic order). Interestingly enough, for massive Euler-Heisenbeg-like electrodynamics (WichmannKroll), we obtain a new long-range $1 / L^{3}$ correction to the interaction energy. Nevertheless, for their noncommutative versions, the static potential becomes ultraviolet finite.

The organization of the paper is as follows: In Section 2, we reexamine Uehling electrodynamics in order to establish a framework for the computation of the static potential. In Section 3 we consider Euler-Heisenberg-like (with a mass term) electrodynamics and show that it yields birefringence, computing the interaction energy for a fermion-antifermion pair and its version in the presence of a minimal length. In Section 4, we repeat our analysis for Euler-Heisenberg electrodynamics in the approximation of the strong-field limit. Finally, in Section 5, we cast our final remarks.

In our conventions, the signature of the metric is $(+1,-1,-1,-1)$.

\section{Brief Review on the Uehling Potential}

As already expressed, we now reexamine the interaction energy for Maxwell theory with an additional term corresponding to the Uehling correction (Uehling electrodynamics). This would not only provide the setup theoretical for our subsequent work, but also fix the notation. To do that we will calculate the expectation value of the energy operator $H$ in the physical state $|\Phi\rangle$, which we will denote by $\langle H\rangle_{\Phi}$. We start off our analysis by considering the effective Lagrangian density [48]:

$$
\mathscr{L}=-\frac{1}{4} F_{\mu \nu}\left(1-\frac{\alpha}{3 \pi} \Delta \mathscr{M}\right) F^{\mu \nu},
$$

where

$$
\mathscr{M}(m, x)=\int_{4 m^{2}}^{\infty} d \tau \frac{1}{\tau(\tau+\Delta)}\left(1+\frac{2 m^{2}}{\tau}\right) \sqrt{1-\frac{4 m^{2}}{\tau}},
$$

with $\Delta \equiv \partial_{\mu} \partial^{\mu}$. It should be noted that $\mathscr{M}$ contains the effect of vacuum polarization to first order in the fine structure constant, $\alpha=e^{2} / \hbar c$, and, $m$ is the electron mass. We may parenthetically note here that the presence of $\Delta$ in (2) does not offer problems. On the one hand, as we will explain below we restrict ourselves to the static case. On the other hand, in order to compute the interaction energy, we will make a series expansion at leading order in $\alpha$.

Before going on, two remarks are pertinent at this point. First, the modification of Coulomb's law in (1) follows from the weak-field limit of the one-loop effective action of quantum electrodynamics (QED). Indeed, as was explained in [48], this modification can be written as

$$
\mathscr{L}_{\mathrm{wf}}=\frac{1}{2} \int A^{\mu}(x) \Pi_{\mu \nu}(x, y) A^{\nu}(y) d^{4} x d^{4} y,
$$

where wf denotes weak field, and $\Pi_{\mu \nu}$ is the usual order $-e^{2}$ polarization tensor of QED. As is well known, in momentum space, this tensor is given by

$$
\Pi_{\mu \nu}(k)=\left(k^{2} g_{\mu \nu}-k_{\mu} k_{\nu}\right) \Pi\left(k^{2}\right),
$$

while the momentum space spectral representation of the polarization function $\Pi\left(k^{2}\right)$ reads

$$
\Pi\left(k^{2}\right)=-\frac{\alpha}{3 \pi} k^{2} \int_{4 m^{2}}^{\infty} d t \frac{\rho(t)}{t} \frac{1}{k^{2}+t},
$$

with $\rho(t)=\left(1+2 m^{2} / t\right) \sqrt{1-4 m^{2} / t}$. Next, due to the tensor structure of $\Pi_{\mu \nu}(k), \mathscr{L}_{\mathrm{wf}}$ can then be expressed in a gaugeinvariant way, that is, in terms of $F_{\mu \nu}$. As a consequence of this, (3) reduces to the modification of Coulomb's law appearing in (1). We mention in passing that in [48] the signature of the metric is different from that used in this paper.

Second, it should be noted that the theory described by (1) contains higher time derivatives; hence to construct the Hamiltonian, one must use, for example, the Ostrogradsky method [49]. Accordingly, in the theory under consideration, the velocities have to be taken as independent canonical 
variables. Let us also mention here that, in previous studies $[50,51]$, we have shown that although theories like (1) contain higher derivatives, in the electrostatic case the canonical momentum conjugate to velocities disappears. Hence the new Legendre transformation to construct the Hamiltonian reduces to the standard Legendre transformation. It should, however, be emphasized here that the present paper is aimed at studying the static potential of the above theory, so that $\Delta$ can be replaced by $-\nabla^{2}$. Notice that, for notational convenience, we have maintained $\Delta$ in (1) and (2), but it should be borne in mind that this paper essentially deals with the static case.

Now, we move on to compute the canonical Hamiltonian. For this end we perform a Hamiltonian constraint analysis. The canonical momenta are found to be $\Pi^{\mu}=$ $(1-(\alpha / 3 \pi) \Delta \mathscr{M}) F^{\mu 0}$. It is easy to see that $\Pi^{0}$ vanishes; we then have the usual constraint equation, which according to Dirac's theory is written as a weak $(\approx)$ equation; that is, $\Pi^{0} \approx 0$. It may be noted that the remaining nonzero momenta must also be written as weak equations. This leads to $\Pi^{i} \approx$ $(1-(\alpha / 3 \pi) \Delta \mathscr{M}) E^{i}$ (with $\left.E_{i}=F_{i 0}\right)$. Accordingly, the canonical Hamiltonian $H_{C}$ is

$$
\begin{aligned}
H_{C} & \approx \int d^{3} x\left[\Pi^{i} \partial_{i} A_{0}-\frac{1}{2} \Pi_{i}\left(1-\frac{\alpha}{3 \pi} \Delta \mathscr{M}\right)^{-1} \Pi^{i}\right. \\
& \left.+\frac{1}{4} F_{i j}\left(1-\frac{\alpha}{3 \pi} \Delta \mathscr{M}\right) F^{i j}\right],
\end{aligned}
$$

which must also be written as a weak equation. Next, the primary constraint, $\Pi^{0} \approx 0$, must be satisfied for all times. An immediate consequence of this is that, using the equation of motion, $\dot{Z} \approx\left[Z, H_{C}\right]$, we obtain the secondary constraint $\Gamma_{1} \equiv \partial_{i} \Pi^{i} \approx 0$, which must also be true for all time. In passing we recall that we are considering the static case; hence this new constraint does not contain time derivatives. It is straightforward to check that there are no further constraints in the theory. Therefore, in the case under consideration, there are two constraints, which are first class. According to the general theory, we obtain the extended Hamiltonian as an ordinary (or strong) equation by adding all the firstclass constraints with arbitrary constraints. We thus write $H=H_{C}+\int d^{3} x\left(u_{0}(x) \Pi_{0}(x)+u_{1}(x) \Gamma_{1}(x)\right)$, where $u_{0}(x)$ and $u_{1}(x)$ are arbitrary Lagrange multipliers. It is also important to observe that when this new Hamiltonian is employed, the equation of motion of a dynamic variable may be written as a strong equation. With the aid of (6), we find that $\dot{A}_{0}(x)=$ $\left[A_{0}(x), H\right]=u_{0}(x)$, which is an arbitrary function. Since $\Pi^{0} \approx 0$ always, neither $A^{0}$ nor $\Pi^{0}$ are of interest in describing the system and may be discarded from the theory. In fact, the term containing $A_{0}$ is redundant, because it can be absorbed by redefining the function $w(x)$. Therefore, the Hamiltonian is now given as

$$
\begin{aligned}
H & =\int d^{3} x\left[w(x) \partial_{i} \Pi^{i}-\frac{1}{2} \Pi_{i}\left(1-\frac{\alpha}{3 \pi} \Delta \mathscr{M}\right)^{-1} \Pi^{i}\right. \\
& \left.+\frac{1}{4} F_{i j}\left(1-\frac{\alpha}{3 \pi} \Delta \mathscr{M}\right) F^{i j}\right],
\end{aligned}
$$

where $w(x)=u_{1}(x)-A_{0}(x)$.
It must be clear from this discussion that the presence of the new arbitrary function, $w(x)$, is undesirable since we have no way of giving it a meaning in a quantum theory. Hence, according to the usual procedure, we impose a gauge condition such that the full set of constraints becomes second class. A convenient choice is

$$
\Gamma_{2}(x) \equiv \int_{C_{\xi x}} d z^{\nu} A_{\nu}(z) \equiv \int_{0}^{1} d \lambda x^{i} A_{i}(\lambda x)=0,
$$

where $\lambda(0 \leq \lambda \leq 1)$ is the parameter describing the spacelike straight path $z^{i}=\xi^{i}+\lambda(x-\xi)^{i}$ and $\xi$ is a fixed point (reference point). There is no essential loss of generality if we restrict our considerations to $\xi^{i}=0$. The Dirac brackets can now be determined and we simply note the only nontrivial Dirac bracket involving the canonical variables; that is,

$$
\begin{aligned}
\left\{A_{i}(x), \Pi^{j}(y)\right\}^{*}= & \delta_{i}^{j} \delta^{(3)}(x-y) \\
& -\partial_{i}^{x} \int_{0}^{1} d \lambda x^{i} \delta^{(3)}(\lambda x-y) .
\end{aligned}
$$

In passing we also recall that the transition to a quantum theory is made by the replacement of the Dirac brackets by the operator commutation relations according to $\{A, B\}^{*} \rightarrow$ $(i / \hbar)[A, B]$.

With the foregoing information, we can now proceed to obtain the interaction energy. As already mentioned, in order to accomplish this purpose, we will calculate the expectation value of the energy operator $H$ in the physical state $|\Phi\rangle$, where the physical states $|\Phi\rangle$ are gauge-invariant ones. The physical state can be written as

$$
\begin{aligned}
|\Phi\rangle & \equiv\left|\bar{\Psi}(\mathbf{y}) \Psi\left(\mathbf{y}^{\prime}\right)\right\rangle \\
& =\bar{\psi}(\mathbf{y}) \exp \left(\frac{i q}{\hbar} \int_{\mathbf{y}^{\prime}}^{\mathbf{y}} d z^{i} A_{i}(z)\right) \psi\left(\mathbf{y}^{\prime}\right)|0\rangle
\end{aligned}
$$

where $|0\rangle$ is the physical vacuum state and the line integral appearing in the above expression is along a space-like path starting at $\mathbf{y}^{\prime}$ and ending at $\mathbf{y}$, on a fixed time slice. The point we wish to emphasize, however, is that the physical fermion $(\Psi(\mathbf{y}))$ is not the Lagrangian fermion $(\psi(\mathbf{y}))$, which is neither gauge-invariant nor associated with an electric field. In fact, the physical fermion is the Lagrangian fermion together with a cloud (or dressing) of gauge fields.

Making use of the above Hamiltonian structure [18], we find that

$$
\begin{aligned}
\Pi_{i}(x)\left|\bar{\Psi}(\mathbf{y}) \Psi\left(\mathbf{y}^{\prime}\right)\right\rangle= & \bar{\Psi}(\mathbf{y}) \Psi\left(\mathbf{y}^{\prime}\right) \Pi_{i}(x)|0\rangle \\
& +q \int_{\mathbf{y}}^{\mathbf{y}^{\prime}} d z_{i} \delta^{(3)}(\mathbf{z}-\mathbf{x})|\Phi\rangle
\end{aligned}
$$

With the aid of (11) and (7), the lowest-order modification in $\alpha$ of the interaction energy takes the form

$$
\langle H\rangle_{\Phi}=\langle H\rangle_{0}+V_{1}+V_{2},
$$


where $\langle H\rangle_{0}=\langle 0|H| 0\rangle$. The $V_{1}, V_{2}$ terms are given by

$$
\begin{aligned}
V_{1} & =\frac{q^{2}}{2} \int d^{3} x \int_{\mathbf{y}^{\prime}}^{\mathbf{y}} d z^{i} \delta^{(3)}(\mathbf{x}-\mathbf{z}) \int_{\mathbf{y}^{\prime}}^{\mathbf{y}} d z^{\prime i} \delta^{(3)}\left(\mathbf{x}-\mathbf{z}^{\prime}\right), \\
V_{2} & =\frac{q^{2}}{2} \frac{\alpha}{3 \pi} \int_{4 m^{2}}^{\infty} d \tau \frac{\rho(\tau)}{\tau} \int d^{3} x \\
& \cdot \int_{\mathbf{y}}^{\mathbf{y}^{\prime}} d z_{i}^{\prime} \delta^{(3)}\left(\mathbf{z}^{\prime}-\mathbf{x}\right) \nabla_{x}^{2} \frac{1}{\tau-\nabla_{x}^{2}} \int_{\mathbf{y}}^{\mathbf{y}^{\prime}} d z^{i} \delta^{(3)}(\mathbf{z}-\mathbf{x}),
\end{aligned}
$$

where $\rho(\tau)=\left(1+2 m^{2} / \tau\right) \sqrt{1-4 m^{2} / \tau}$.

We note that term (13) may look peculiar, but it is nothing but the familiar Coulomb interaction plus a self-energy term [52]. Now making use of the Green function, $G\left(\mathbf{z}, \mathbf{z}^{\prime}\right)=$ $(1 / 4 \pi)\left(e^{-\sqrt{\tau}\left|\mathbf{z}-\mathbf{z}^{\prime}\right|} /\left|\mathbf{z}-\mathbf{z}^{\prime}\right|\right)$, the term (14) can be rewritten in the form

$$
\begin{aligned}
V_{2} & =\frac{q^{2}}{2} \frac{\alpha}{3 \pi} \int_{4 m^{2}}^{\infty} d \tau \frac{\rho(\tau)}{\tau} \int_{\mathbf{y}}^{\mathbf{y}^{\prime}} d z_{i}^{\prime} \nabla_{z^{\prime}}^{2} \int_{\mathbf{y}}^{\mathbf{y}^{\prime}} d z^{i} G\left(\mathbf{z}, \mathbf{z}^{\prime}\right) \\
& =-\frac{\alpha}{3 \pi} \frac{q^{2}}{2} \int_{4 m^{2}}^{\infty} d \tau \frac{\rho(\tau)}{\tau} \frac{e^{-\sqrt{\tau}\left|\mathbf{y}-\mathbf{y}^{\prime}\right|}}{\left|\mathbf{y}-\mathbf{y}^{\prime}\right|} .
\end{aligned}
$$

Since the second and third term on the right-hand side of (12) are clearly dependent on the distance between the external static fields, the potential for two opposite charges located at $\mathbf{y}$ and $\mathbf{y}^{\prime}$ reads

$$
V=-\frac{q^{2}}{4 \pi} \frac{1}{L}\left(1+\frac{\alpha}{3 \pi} \int_{4 m^{2}}^{\infty} d \tau \frac{\rho(\tau)}{\tau} e^{-\sqrt{\tau} L}\right)
$$

where $L=\left|\mathbf{y}-\mathbf{y}^{\prime}\right|$. Accordingly, one recovers the known Uehling potential, which finds here an entirely different derivation.

Before we proceed further, we wish to show that this result can be written alternatively in a more explicit form. Making use of [53]

$$
\chi_{n}(z)=\int_{1}^{\infty} d t \frac{e^{-t z}}{t^{n}}\left(1+\frac{1}{2 t^{2}}\right) \sqrt{1-\frac{1}{t^{2}}}
$$

we then get

$$
V=-\frac{q^{2}}{4 \pi} \frac{1}{L}\left(1+\frac{2 \alpha}{3 \pi} \chi_{1}(2 m L)\right) .
$$

By the transformation, $t=\cosh u$ [54], the functions $\chi_{n}$ can be reduced to the form [53]

$$
\chi_{n}(z)=K i_{n-1}(z)-\frac{1}{2} K i_{n+1}(z)-\frac{1}{2} K i_{n+3}(z)
$$

where the functions $K i$ denote Bessel function integrals. Hence we see that the interaction energy (with $m=1$ ) becomes

$$
\begin{aligned}
V & =-\frac{q^{2}}{4 \pi} \frac{1}{L}\left\{1+\frac{2 \alpha}{3 \pi}\left[\left(1+\frac{L^{2}}{3}\right) K_{0}(2 L)\right.\right. \\
& -\left(\frac{5 L}{3}+\frac{2 L^{3}}{3}\right) K_{1}(2 L) \\
& \left.\left.+\left(\frac{3 L}{2}+\frac{2 L^{3}}{3}\right) \int_{2 L}^{\infty} d t K_{0}(t)\right]\right\},
\end{aligned}
$$

where $K_{0}(z)$ and $K_{1}(z)$ are modified Bessel functions. Finally, with the aid of asymptotic forms for Bessel functions, it is a simple matter to find expressions for $V$ for large and small $L$.

Before concluding this subsection, we discuss an alternative way of stating our previous result (16), which displays certain distinctive features of our methodology. We start by considering $[52,55]$

$$
V \equiv q\left(\mathscr{A}_{0}(\mathbf{0})-\mathscr{A}_{0}(\mathbf{L})\right)
$$

where the physical scalar potential is given by

$$
\mathscr{A}_{0}(t, \mathbf{r})=\int_{0}^{1} d \lambda r^{i} E_{i}(t, \lambda \mathbf{r}) .
$$

This follows from the vector gauge-invariant field expression:

$$
\mathscr{A}_{\mu}(x) \equiv A_{\mu}(x)+\partial_{\mu}\left(-\int_{\xi}^{x} d z^{\mu} A_{\mu}(z)\right),
$$

where the line integral is along a space-like path from the point $\xi$ to $x$, on a fixed slice time. It is also important to observe that the gauge-invariant variables (22) commute with the sole first constraint (Gauss law), showing in this way that these fields are physical variables. In as much as we are interested in estimating the lowest-order correction to the Coulomb energy, we will retain only the leading term in expression $E^{i}=(1-(\alpha / 3 \pi) \Delta \mathscr{M})^{-1} \Pi^{i}$. Making use of this last expression, (22) gives

$$
\begin{aligned}
& \mathscr{A}_{0}(t, \mathbf{r}) \\
& =\int_{0}^{1} d \lambda r^{i} \partial_{i}^{\lambda \mathbf{r}}\left(-\frac{J^{0}(\lambda \mathbf{r})}{\nabla_{\lambda \mathbf{r}}^{2}}\right) \\
& \quad+\frac{\alpha}{3 \pi} \int_{4 m^{2}}^{\infty} \frac{d \tau}{\tau} \rho(\tau) \int_{0}^{1} d \lambda r^{i} \partial_{i}^{\lambda \mathbf{r}}\left(-\frac{J^{0}(\lambda \mathbf{r})}{\nabla_{\lambda \mathbf{r}}^{2}-\tau}\right) ;
\end{aligned}
$$

to get the last line we used Gauss law for the present theory; that is, $\partial_{i} \Pi^{i}=J^{0}$ (where we have included the external current $J^{0}$ to represent the presence of two opposite charges). Accordingly, for $J^{0}(t, \mathbf{r})=q \delta^{(3)}(\mathbf{r})$, the potential for a pair of static point-like opposite charges located at $\mathbf{0}$ and $\mathbf{L}$ is given by

$$
V=-\frac{q^{2}}{4 \pi} \frac{1}{L}\left(1+\frac{\alpha}{3 \pi} \int_{4 m^{2}}^{\infty} d \tau \frac{\rho(\tau)}{\tau} e^{-\sqrt{\tau} L}\right)
$$

after subtracting a self-energy term. 


\section{Euler-Heisenberg-Like Model}

Proceeding in the same way as we did in the foregoing section, we will now consider the interaction energy for EulerHeisenberg-like electrodynamics. Nevertheless, in order to put our discussion into context, it is useful to describe very briefly the model under consideration. In such a case, the Lagrangian density reads

$$
\mathscr{L}=\frac{\beta^{2}}{2}\left\{1-\left[1+\frac{1}{\beta^{2}} \mathscr{F}-\frac{1}{\beta^{2} \gamma^{2}} \mathscr{G}^{2}\right]^{p}\right\},
$$

where we have included two parameters: $\beta$ and $\gamma$. As usual, $\mathscr{F}=(1 / 4) F_{\mu \nu} F^{\mu \nu}, \mathscr{G}=(1 / 4) F_{\mu \nu} \widetilde{F}^{\mu \nu}, F_{\mu \nu}=\partial_{\mu} A_{\nu}-\partial_{\nu} A_{\mu}$, and $\widetilde{F}^{\mu \nu}=(1 / 2) \varepsilon^{\mu \nu \rho \lambda} F_{\rho \lambda}$. Let us also mention here that in our previous paper [19] we have studied the domain $0<$ $p<1$. Moreover, it follows from (26) that when $p=2$ the model contains, to orders $\mathcal{O}\left(1 / \beta^{2}\right)$ and $\mathcal{O}\left(1 / \gamma^{2}\right)$, a EulerHeisenberg-like model with the appropriate identifications of the constants. Interestingly, we also observe that in the limit $\gamma \rightarrow \infty$ we obtain a Wichmann-Kroll model. This remark opens up the way to discuss the effect of these nonlinear corrections on the interaction energy, as we are going to study below. In fact, we will consider a massive WichmannKroll system. The motivation for this study comes from recent considerations in the context of dualities [56], where massive Born-Infeld systems play an important role.

Having made these observations, we can write immediately the field equations for $p=2$,

$$
\partial_{\mu}\left[\Gamma\left(F^{\mu \nu}-\frac{2}{\gamma^{2}} \mathscr{G} \widetilde{F}^{\mu \nu}\right)\right]=0,
$$

while the Bianchi identities are given by

$$
\partial_{\mu} \widetilde{F}^{\mu \nu}=0
$$

where

$$
\Gamma=1+\frac{\mathscr{F}}{\beta^{2}}-\frac{\mathscr{G}^{2}}{\beta^{2} \gamma^{2}} .
$$

Also, it is straightforward to see that Gauss law becomes

$$
\nabla \cdot \mathbf{D}=0
$$

where $\mathbf{D}$ is given by

D

$$
=\left[1-\frac{\left(\mathbf{E}^{2}-\mathbf{B}^{2}\right)}{2 \beta^{2}}-\frac{(\mathbf{E} \cdot \mathbf{B})^{2}}{\beta^{2} \gamma^{2}}\right]\left(\mathbf{E}+\frac{2}{\gamma^{2}}(\mathbf{E} \cdot \mathbf{B}) \mathbf{B}\right) .
$$

Again, from (30), for $J^{0}(t, \mathbf{r})=e \delta^{(3)}(\mathbf{r})$, we find $\mathbf{D}=\left(Q / r^{2}\right) \widehat{r}$, where $Q=e / 4 \pi$. This then implies that, for a point-like charge, $e$, at the origin, the expression

$$
\frac{Q}{r^{2}}=\left(1-\frac{\mathbf{E}^{2}}{2 \beta^{2}}\right)|\mathbf{E}|
$$

tells us that, for $r \rightarrow 0$, the electrostatic field becomes singular at $r=0$, in contrast to the $0<p<1$ case where the electrostatic field is finite. Even so, in this theory the phenomenon of birefringence is present. Before going into details, we would like to recall that birefringence refers to the property that polarized light in a particular direction (optical axis) travels at a different velocity from that of light polarized in a direction perpendicular to this axis. Indeed, due to quantum fluctuations, the QED vacuum has this property, as we are going to show.

To illustrate this important feature, we introduce the vectors $\mathbf{D}=\partial \mathscr{L} / \partial \mathbf{E}$ and $\mathbf{H}=-\partial \mathscr{L} / \partial \mathbf{B}$ :

$$
\begin{aligned}
& \mathbf{D}=\Gamma\left(\mathbf{E}+2 \frac{\mathbf{B}(\mathbf{E} \cdot \mathbf{B})}{\gamma^{2}}\right), \\
& \mathbf{H}=\Gamma\left(\mathbf{B}-2 \frac{\mathbf{E}(\mathbf{E} \cdot \mathbf{B})}{\gamma^{2}}\right),
\end{aligned}
$$

where $\Gamma=1+\left(1 / 2 \beta^{2}\right)\left(\mathbf{B}^{2}-\mathbf{E}^{2}\right)-\left(1 / \beta^{2} \gamma^{2}\right)(\mathbf{E} \cdot \mathbf{B})^{2}$. We thus obtain the equations of motion

$$
\begin{array}{r}
\nabla \cdot \mathbf{D}=0 \\
\frac{\partial \mathbf{D}}{\partial t}-\nabla \times \mathbf{H}=0 \\
\nabla \cdot \mathbf{B}=0, \\
\frac{\partial \mathbf{B}}{\partial t}+\nabla \times \mathbf{E}=0 .
\end{array}
$$

With the aid from (33), we find the electric permittivity, $\varepsilon_{i j}$, and the inverse magnetic permeability, $\left(\mu^{-1}\right)_{i j}$, tensors of the vacuum; that is,

$$
\begin{aligned}
\varepsilon_{i j} & =\Gamma\left(\delta_{i j}+\frac{2 B_{i} B_{j}}{\gamma^{2}}\right), \\
\left(\mu^{-1}\right)_{i j} & =\Gamma\left(\delta_{i j}-\frac{2 E_{i} E_{j}}{\gamma^{2}}\right),
\end{aligned}
$$

with $D_{i}=\varepsilon_{i j} E_{j}$ and $B_{i}=\mu_{i j} H_{j}$.

In accordance with our previous procedure [18, 19], we can now linearize the above equations. To do this, it is advantageous to introduce a weak electromagnetic wave $\left(\mathbf{E}_{p}, \mathbf{B}_{p}\right)$ propagating in the presence of a strong constant external field $\left(\mathbf{E}_{0}, \mathbf{B}_{0}\right)$. On these assumptions, we readily find that, for the case of a purely magnetic field $\left(\mathbf{E}_{0}=0\right)$, the vectors $\mathbf{D}$ and $\mathbf{H}$ become

$$
\begin{aligned}
\mathbf{D} & =\left(1+\frac{\mathbf{B}_{0}^{2}}{2 \beta^{2}}\right)\left[\mathbf{E}_{p}+\frac{2}{\gamma^{2}}\left(\mathbf{E}_{p} \cdot \mathbf{B}_{0}\right) \mathbf{B}_{0}\right], \\
\mathbf{H} & =\left(1+\frac{\mathbf{B}_{0}^{2}}{2 \beta^{2}}\right) \\
& \cdot\left[\mathbf{B}_{p}+\frac{1}{\beta^{2}\left(1+\mathbf{B}_{0}^{2} / 2 \beta^{2}\right)}\left(\mathbf{B}_{p} \cdot \mathbf{B}_{0}\right) \mathbf{B}_{0}\right],
\end{aligned}
$$

where we have to keep only linear terms in $\mathbf{E}_{p}, \mathbf{B}_{p}$. As before, we consider the $z$-axis as the direction of the external 
magnetic field ( $\mathbf{B}_{0}=B_{0} \mathbf{e}_{3}$ ), and, assuming that the light wave moves along the $x$-axis, the decomposition into a plane wave for the fields $\mathbf{E}_{p}$ and $\mathbf{B}_{p}$ can be written as

$$
\begin{aligned}
& \mathbf{E}_{p}(\mathbf{x}, t)=\mathbf{E} e^{-i(w t-\mathbf{k} \cdot \mathbf{x})}, \\
& \mathbf{B}_{p}(\mathbf{x}, t)=\mathbf{B} e^{-i(w t-\mathbf{k} \cdot \mathbf{x})} .
\end{aligned}
$$

In this case, it clearly follows that

$$
\begin{aligned}
& \left(\frac{k^{2}}{w^{2}}-\varepsilon_{22} \mu_{33}\right) E_{2}=0, \\
& \left(\frac{k^{2}}{w^{2}}-\varepsilon_{33} \mu_{22}\right) E_{3}=0 .
\end{aligned}
$$

As a consequence, we have two different situations. First, if $\mathbf{E} \perp \mathbf{B}_{0}$ (perpendicular polarization), from (39) $E_{3}=0$, and from (38) we get $k^{2} / w^{2}=\varepsilon_{22} \mu_{33}$. This then means that the dispersion relation of the photon takes the form

$$
n_{\perp}=\sqrt{\frac{1+\mathbf{B}_{0}^{2} / 2 \beta^{2}}{1+3 \mathbf{B}_{0}^{2} / 2 \beta^{2}}} .
$$

Second, if $\mathbf{E} \| \mathbf{B}_{0}$ (parallel polarization), from (38) $E_{2}=0$, and from (39) we get $k^{2} / w^{2}=\varepsilon_{33} \mu_{22}$. This leads to

$$
n_{\|}=\sqrt{1+\frac{2 \mathbf{B}_{0}^{2}}{\gamma^{2}}}
$$

Thus we verify that in the case of a generalized EulerHeisenberg electrodynamics the phenomenon of birefringence is present.

We now pass to the calculation of the interaction energy between static point-like sources for a massive WichmannKroll-like model; our analysis follows closely that of $[18,19]$. The corresponding theory is governed by the Lagrangian density:

$$
\mathscr{L}=-\frac{1}{4} F_{\mu \nu}^{2}+\frac{1}{32 \beta^{2}}\left(F_{\mu \nu} F^{\mu \nu}\right)^{2}+\frac{m^{2}}{2} A_{\mu} A^{\mu} .
$$

Next, in order to handle the second term on the right hand in (42), we introduce an auxiliary field $\xi$ such that its equation of motion gives back the original theory. This allows us to write the Lagrangian density as

$$
\begin{aligned}
\mathscr{L}= & -\frac{1}{4} F_{\mu \nu} F^{\mu \nu}+\frac{\xi}{32 \beta^{2}} F_{\mu \nu} F^{\mu \nu}-\frac{1}{128 \beta^{2}} \xi^{2} \\
& +\frac{m^{2}}{2} A_{\mu} A^{\mu} .
\end{aligned}
$$

With the redefinition $\eta=1-\xi / 8 \beta^{2}$, (43) becomes

$$
\mathscr{L}=-\frac{1}{4} \eta F_{\mu \nu} F^{\mu \nu}-\frac{1}{2}(1-\eta)^{2}+\frac{m^{2}}{2} A_{\mu} A^{\mu} .
$$

Before we proceed to work out explicitly the interaction energy, we will first restore the gauge invariance in (44).
Following an earlier procedure, we readily verify that the canonical momenta read $\Pi^{\mu}=-\eta F^{0 \mu}$, which results in the usual primary constraint $\Pi^{0}=0$, and $\Pi^{i}=\eta F^{i 0}$. In this way one obtains

$$
\begin{aligned}
H_{C} & =\int d^{3} x\left\{\Pi^{i} \partial_{i} A_{0}+\frac{1}{2 \eta} \Pi^{2}+\frac{\eta}{2} \mathbf{B}^{2}-\frac{m^{2}}{2} A_{\mu} A^{\mu}\right. \\
& \left.+\frac{1}{2}(1-\eta)^{2}\right\} .
\end{aligned}
$$

The consistency condition, $\dot{\Pi}=0$, leads to the constraint $\Gamma \equiv$ $\partial_{i} \Pi^{i}+m^{2} A^{0}=0$. As a result, both constraints are second-class. To convert the second-class system into first-class, we will adopt the procedure described previously. Thus, we enlarge the original phase space by introducing a canonical pair of fields $\theta$ and $\Pi_{\theta}$. It follows, therefore, that a new set of firstclass constraints can be defined in this extended space: $\Lambda_{1} \equiv$ $\Pi_{0}+m^{2} \theta=0$ and $\Lambda_{2} \equiv \Gamma+\Pi_{\theta}=0$. Notice that this new $\theta$-field is not to be confused with the usual noncommutative parameter. This then shows that the new constraints are first-class and, therefore, restore the gauge symmetry. As is well known, this procedure reproduces the usual Stückelberg formalism. From this, the new effective Lagrangian density, after integrating out the $\theta$ fields, becomes

$$
\mathscr{L}=-\frac{1}{4} F_{\mu \nu}\left(\eta+\frac{m^{2}}{\Delta}\right) F^{\mu \nu}-\frac{1}{2}(1-\eta)^{2} .
$$

Now, writing $\sigma=\eta+m^{2} / \Delta$, expression (46) can be brought to the form

$$
\mathscr{L}=-\frac{1}{4} F_{\mu \nu} \sigma F^{\mu \nu}-\frac{k}{128}\left(1-\sigma+\frac{m^{2}}{\Delta}\right)^{2},
$$

where $k=64 \beta^{2}$.

We are now ready to compute the interaction energy. In this case, the canonical momenta are $\Pi^{\mu}=-\sigma F^{0 \mu}$, with the usual primary constraint $\Pi^{0}=0$, and $\Pi^{i}=\sigma F^{i 0}$. Hence the canonical Hamiltonian is expressed as

$$
\begin{aligned}
H_{C} & =\int d^{3} x\left\{\Pi^{i} \partial_{i} A_{0}+\frac{1}{2 \sigma} \Pi^{2}+\frac{\sigma}{2} \mathbf{B}^{2}\right. \\
& \left.+\frac{k}{128}\left(1-\sigma+\frac{m^{2}}{\Delta}\right)^{2}\right\}
\end{aligned}
$$

Time conservation of the primary constraint $\Pi^{0}$ yields the secondary constraint $\Gamma_{1} \equiv \partial_{i} \Pi^{i}=0$. Similarly the $\mathscr{P}_{\sigma}$ constraint yields no further constraints and just determines the field $\sigma$. In this case, at leading order in $\beta$, the field $\sigma$ is given by

$$
\begin{aligned}
\sigma= & \left(1+\frac{m^{2}}{\Delta}-\frac{\mathbf{B}^{2}}{2 \beta^{2}}\right) \\
& \cdot\left[1-\frac{3}{2 \beta^{2}} \frac{1}{\left(1+m^{2} / \Delta-\mathbf{B}^{2} / 2 \beta^{2}\right)^{3}} \boldsymbol{\Pi}^{2}\right],
\end{aligned}
$$


which will be used to eliminate $\sigma$. As before, the corresponding total (first-class) Hamiltonian that generates the time evolution of the dynamical variables is $H=H_{C}+$ $\int d^{3} x\left(u_{0}(x) \Pi_{0}(x)+u_{1}(x) \Gamma_{1}(x)\right)$, where $u_{0}(x)$ and $u_{1}(x)$ are the Lagrange multiplier utilized to implement the constraints.

In the same way as was done in the previous subsection, the expectation value of the energy operator $H$ in the physical state $|\Phi\rangle$ becomes

$$
\begin{aligned}
& \langle H\rangle_{\Phi}=\langle\Phi| \int d^{3} x\left\{\frac{1}{2} \Pi^{i}\left(1+\frac{m^{2}}{\Delta}\right)^{-1} \Pi^{i}+\frac{15}{8 \beta^{2}} \Pi^{4}\right. \\
& \left.-\frac{15 m^{2}}{2 \beta^{2}} \Pi^{2} \frac{1}{\Delta} \Pi^{2}\right\}|\Phi\rangle ;
\end{aligned}
$$

in this last line we have considered only quadratic terms in $m^{2}$.

In such a case, by employing (50), the lowest-order modification in $\beta^{2}$ and $m^{2}$ of the interaction energy takes the form

$$
\langle H\rangle_{\Phi}=\langle H\rangle_{0}+V_{1}+V_{2}+V_{3},
$$

where $\langle H\rangle_{0}=\langle 0|H| 0\rangle$. The $V_{1}, V_{2}$, and $V_{3}$ terms are given by

$$
\begin{aligned}
V_{1} & =\frac{q^{2}}{2} \int d^{3} x \int_{\mathbf{y}^{\prime}}^{\mathbf{y}} d z^{\prime i} \delta^{(3)}\left(\mathbf{x}-\mathbf{z}^{\prime}\right)\left(1-\frac{m^{2}}{\nabla^{2}}\right)^{-1} \\
& \cdot \int_{\mathbf{y}^{\prime}}^{\mathbf{y}} d z^{i} \delta^{(3)}(\mathbf{x}-\mathbf{z}), \\
V_{2}= & -\frac{15 q^{4}}{8 \beta^{2}} \int d^{3} x \int_{\mathbf{y}^{\prime}}^{\mathbf{y}} d z_{i} \delta^{(3)}(\mathbf{x}-\mathbf{z}) \\
& \cdot \int_{\mathbf{y}^{\prime}}^{\mathbf{y}} d z^{\prime i} \delta^{(3)}\left(\mathbf{x}-\mathbf{z}^{\prime}\right) \int_{\mathbf{y}^{\prime}}^{\mathbf{y}} d u^{k} \delta^{(3)}(\mathbf{x}-\mathbf{u}) \\
& \cdot \int_{\mathbf{y}^{\prime}}^{\mathbf{y}} d v_{k} \delta^{(3)}(\mathbf{x}-\mathbf{v}), \\
V_{3} & =\frac{15 m^{2} q^{4}}{2 \beta^{2}} \int d^{3} x \int_{\mathbf{y}^{\prime}}^{\mathbf{y}} d z_{i} \delta^{(3)}(\mathbf{x}-\mathbf{z}) \\
& \cdot \int_{\mathbf{y}^{\prime}}^{\mathbf{y}} d z^{\prime i} \delta^{(3)}\left(\mathbf{x}-\mathbf{z}^{\prime}\right) \int_{\mathbf{y}^{\prime}}^{\mathbf{y}} d u^{k} \delta^{(3)}(\mathbf{x}-\mathbf{u}) \\
& \cdot \int_{\mathbf{y}^{\prime}}^{\mathbf{y}} d v_{k} \delta^{(3)}(\mathbf{x}-\mathbf{v}) .
\end{aligned}
$$

Finally, with the aid of expressions (52), the potential for a pair of static point-like opposite charges located at $\mathbf{0}$ and $\mathbf{L}$ is given by

$$
V=-\frac{q^{2}}{4 \pi} \frac{e^{-m L}}{L}+\frac{q^{4}}{16 \pi \beta^{2}}\left(\frac{3}{8 \pi} \frac{1}{L^{2}}-5 m^{2}\right) \frac{1}{L^{3}}
$$

observe that when $m=0$, profile (53) reduces to the known Wichmann-Kroll interaction energy. On the other hand, for $m \neq 0$, the key role played by the mass term in transforming the Coulomb potential into the Yukawa one should be noted. Interestingly enough, an unexpected feature is found. In fact, profile (53) displays a new long-range $1 / L^{3}$ correction, where its strength is proportional to $\mathrm{m}^{2}$. It is also important to observe that an analogous correction has been found in BornInfeld electrodynamics in the context of very special relativity [57]. In this way, we establish a new connection between nonlinear effectives theories.

Before we proceed further, we should comment on our result. In the case of QED (Euler-Heisenberg Lagrangian density), the parameter $1 / \beta^{2}$ is given by $1 / \beta^{2}=(16 / 45)\left(e^{4} \hbar /\right.$ $m_{e}^{4} c^{7}$ ), where $m_{e}$ is the electron mass. In this context, we also recall the currently accepted upper limit for the photon mass; that is, $m_{\gamma} \sim 2 \times 10^{-16} \mathrm{eV}$. Thus, for the QED case, from (53) it follows that the second term on the right hand side would be detectable in long-range distances $\left(\sim 10^{9} \mathrm{~m}\right)$. In other words, we see that detectable corrections induced by vacuum polarization with a mass term would be present at low energy scales.

From (53), it clearly follows that the interaction energy between heavy charged charges, at leading order in $\beta$, is not finite at the origin. Motivated by this, one may consider the above calculation in a noncommutative geometry, based on findings of our previous studies $[18,19]$. In such a case, the electric field at leading order in $\beta^{2}$ and $m^{2}$ takes the form

$$
\begin{aligned}
E_{i}= & {\left[\left(1+\frac{m^{2}}{\Delta}\right)^{-1}+\frac{3}{2 \beta^{2}} \Pi^{2}-\frac{6 m^{2}}{\beta^{2}} \Pi^{2} \frac{1}{\Delta}\right] } \\
& \cdot \partial_{i}\left(-\frac{e^{\theta \nabla^{2}} \delta^{(3)}(\mathbf{x})}{\nabla^{2}}\right),
\end{aligned}
$$

where it may be recalled that we are now replacing the source $\delta^{(3)}(\mathbf{x}-\mathbf{y})$ by the smeared source $e^{\theta \nabla^{2}} \delta^{(3)}(\mathbf{x}-\mathbf{y})$, with the parameter $\theta$ being noncommutative. Now, making use of (22), we readily find that

$$
\mathscr{A}_{0}(t, \mathbf{r})=\mathscr{A}_{0}^{(1)}(t, \mathbf{r})+\mathscr{A}_{0}^{(2)}(t, \mathbf{r})+\mathscr{A}_{0}^{(3)}(t, \mathbf{r}) .
$$

The term $\mathscr{A}_{0}^{(1)}(t, \mathbf{r})$ was first calculated in [50]; we can, therefore, write only the result:

$$
\begin{aligned}
& \mathscr{A}_{0}^{(1)}(t, \mathbf{r}) \\
& =q \frac{e^{m^{2} \theta}}{4 \pi} \frac{1}{r}\left[e^{-m r}-\frac{1}{\sqrt{\pi}} \int_{r^{2} / 4 \theta}^{\infty} d u \frac{1}{\sqrt{u}} e^{-u-m^{2} r^{2} / 4 u}\right] \\
& \quad-q \frac{m}{4 \pi} e^{m^{2} \theta} .
\end{aligned}
$$


Meanwhile, the terms $\mathscr{A}_{0}^{(2)}(t, \mathbf{r})$ and $\mathscr{A}_{0}^{(3)}(t, \mathbf{r})$, after some manipulation, can be brought to the form

$$
\begin{gathered}
\mathscr{A}_{0}^{(2)}(t, \mathbf{r})=\frac{12 q^{3}}{\beta^{2} \pi^{3 / 2}} \widehat{n}^{i} \int_{0}^{x} d u^{i} \frac{1}{u^{6}} \gamma^{3}\left(\frac{3}{2}, \frac{u^{2}}{4 \theta}\right), \\
\mathscr{A}_{0}^{(3)}(t, \mathbf{r})=\frac{3 m^{2} q^{3}}{\beta^{2} \pi^{5 / 2}} \widehat{n}^{i} \int_{0}^{x} d u^{i} \frac{1}{u^{4}} \gamma^{2}\left(\frac{3}{2}, \frac{u^{2}}{4 \theta}\right) \\
\cdot\left[\frac{4 \theta}{u^{2}} \gamma\left(\frac{3}{2}, \frac{u^{2}}{4 \theta}\right)-\gamma\left(\frac{1}{2}, \frac{u^{2}}{4 \theta}\right)\right],
\end{gathered}
$$

where $\gamma\left(3 / 2, r^{2} / 4 \theta\right)$ is the lower incomplete Gamma function defined by $\gamma(a / b, x) \equiv \int_{0}^{x}(d u / u) u^{a / b} e^{-u}$.

Inserting these expressions in (21), we finally obtain the static potential for two opposite charges $q$ located at $\mathbf{0}$ and $\mathbf{L}$ as

$$
\begin{aligned}
V=- & \frac{q}{4 \pi} \frac{e^{m^{2} \theta}}{L}\left[e^{-m L}-\frac{1}{\sqrt{\pi}} \int_{L^{2} / 4 \theta}^{\infty} d u \frac{1}{\sqrt{u}} e^{-u-m^{2} u^{2} / 4 u}\right] \\
- & \frac{12 q^{4}}{\beta^{2} \pi^{3 / 2}} \widehat{n}^{i} \int_{0}^{L} d u^{i} \frac{1}{u^{6}} \gamma^{3}\left(\frac{3}{2}, \frac{u^{2}}{4 \theta}\right)-\frac{3 m^{2} q^{4}}{\beta^{2} \pi^{5 / 2}} \\
& \cdot \hat{n}^{i} \int_{0}^{x} d u^{i} \frac{1}{u^{4}} \gamma^{2}\left(\frac{3}{2}, \frac{u^{2}}{4 \theta}\right) \\
& \cdot\left[\frac{4 \theta}{u^{2}} \gamma\left(\frac{3}{2}, \frac{u^{2}}{4 \theta}\right)-\gamma\left(\frac{1}{2}, \frac{u^{2}}{4 \theta}\right)\right],
\end{aligned}
$$

which is finite for $L \rightarrow 0$. It is a simple matter to verify that in the limit $\theta \rightarrow 0$ we recover our above result.

\section{Logarithmic Correction}

We now want to extend what we have done to EulerHeisenberg-like electrodynamics at strong fields. As already mentioned, such theories show a power behavior that is typical for critical phenomena [58]. In such a case, the Lagrangian density reads

$$
\mathscr{L}=-\frac{1}{4} F_{\mu \nu} F^{\mu \nu}-\frac{b_{0}}{8} F_{\mu \nu} F^{\mu \nu} \log \left(\frac{F_{\mu \nu} F^{\mu \nu}}{4 \lambda^{2}}\right),
$$

where $b_{0}$ and $\lambda$ are constants. In fact, by choosing $b_{0}=$ $e^{2} / 6 \pi^{2}$ and $\lambda=m_{e}^{2} c^{3} / e \hbar$, we recover the Euler-Heisenberg electrodynamics at strong fields [58].

In the same way as was done in the previous section, one can introduce an auxiliary field, $\xi$, to handle the logarithm in (59). This leads to

$$
\mathscr{L}=-\frac{1}{4} \alpha_{1} F_{\mu \nu} F^{\mu \nu}-\alpha_{2}\left(F_{\mu \nu} F^{\mu \nu}\right)^{2}
$$

where $\alpha_{1}=1-\left(b_{0} / 2\right)(1+\log \xi)$ and $\alpha_{2}=b_{0} \xi / 32 \lambda^{2}$.

A similar procedure can be used to manipulate the quadratic term in (60). Accordingly, by introducing a second auxiliary field, $\eta$, one easily finds

$$
\mathscr{L}=-\frac{1}{4} F_{\mu \nu}\left(\alpha_{1}+4 \alpha_{2} \eta\right) F^{\mu \nu}+\frac{\eta^{2}}{4} \alpha_{2} .
$$

By setting, $\sigma=\alpha_{1}+4 \alpha_{2} \eta$, we then have

$$
\mathscr{L}=-\frac{1}{4} \sigma F_{\mu \nu} F^{\mu \nu}+\frac{1}{64 \alpha_{2}}\left(\sigma-\alpha_{1}\right)^{2} .
$$

It is once again straightforward to apply the gaugeinvariant formalism discussed in the foregoing section. The canonical momenta read $\Pi^{\mu}=-\sigma F^{0 \mu}$, and at once we recognize the two primary constraints $\Pi^{0}=0$ and $\mathscr{P}_{\sigma} \equiv$ $\partial L / \partial \dot{\sigma}=0$. The canonical Hamiltonian corresponding to (62) is

$$
\begin{aligned}
H_{C} & =\int d^{3} x \\
& \cdot\left\{\Pi_{i} \partial^{i} A_{0}+\frac{1}{2 \sigma} \Pi^{2}+\frac{\sigma}{2} \mathbf{B}^{2}-\frac{1}{64 \alpha_{2}}\left(\sigma-\alpha_{1}\right)^{2}\right\} .
\end{aligned}
$$

Requiring the primary constraint $\Pi^{0}$ to be preserved in time, one obtains the secondary constraint $\Gamma_{1}=\partial_{i} \Pi^{i}=0$. In the same way, for the constraint $\mathscr{P}_{\sigma}$, we get the auxiliary field $\sigma$ as

$$
\begin{aligned}
\sigma= & \left(1-\frac{b_{0}}{2}(1+\ln \xi)+\frac{b_{0} \mathbf{B}^{2}}{2 \lambda^{2}} \xi\right)[1 \\
& \left.+\frac{3 b_{0} \mathbf{B}^{2}}{2 \lambda^{2}} \frac{\xi}{\left(1-\left(b_{0} / 2\right)(1+\ln \xi)+\left(b_{0} \mathbf{B}^{2} / 2 \lambda^{2}\right) \xi\right)^{3}}\right] .
\end{aligned}
$$

Hence we obtain

$$
\begin{aligned}
& H_{C}=\int d^{3} x \\
& \quad \cdot\left\{\Pi_{i} \partial^{i} A_{0}+\frac{1}{2} \Pi^{2}+\frac{b_{0}}{4}(1+\log \xi) \Pi^{2}-\frac{3 b_{0} \xi}{2 \lambda^{2}} \Pi^{4}\right\} .
\end{aligned}
$$

As before, requiring the primary constraint $\mathscr{P}_{\xi}$ to be preserved in time, one obtains the auxiliary field $\xi$. In this case $\xi=\lambda / 6 \Pi^{2}$. Consequently, we get

$$
H_{C}=\int d^{3} x\left\{\Pi_{i} \partial^{i} A_{0}+\frac{1}{2}\left(1+b_{0}\right) \Pi^{2}-\frac{6 b_{0}}{\lambda^{2}} \Pi^{4}\right\} .
$$

Following the same steps that led to (50) we find that

$$
\langle H\rangle_{\Phi}^{(1)}=\left\langle\Phi\left|\int d^{3} x\left\{\frac{1}{2} \Pi^{2}-\frac{3}{8 \beta^{2}} \Pi^{4}\right\}\right| \Phi\right\rangle .
$$

It should be noted that this expression is similar to (50) in the limit $m \rightarrow 0$, except by the changed sign in front of the $\Pi^{4}$ term. Hence we see that the potential for two opposite charges in $\mathbf{0}$ and $\mathbf{L}$ is given by

$$
V=-\frac{q^{2}}{4 \pi} \frac{1}{L}-\frac{q^{4}}{6040 \beta^{2} \pi^{2}} \frac{1}{L^{5}} .
$$

\section{Final Remarks}

Finally, within the gauge-invariant but path-dependent variables formalism, we have considered the confinement versus 
screening issue for both massive Euler-Heisenberg-like and Euler-Heisenberg electrodynamics in the approximation of the strong-field limit. Once again, a correct identification of physical degrees of freedom has been fundamental for understanding the physics hidden in gauge theories. Interestingly enough, their noncommutative version displays an ultraviolet finite static potential. The analysis above reveals the key role played by the new quantum of length in our analysis. In a general perspective, the benefit of considering the present approach is to provide a unification scenario among different models as well as exploiting the equivalence in explicit calculations, as we have illustrated in the course of this work.

\section{Conflict of Interests}

The author declares that there is no conflict of interests regarding the publication of this paper.

\section{Acknowledgments}

It is a pleasure for the author to thank J. A. Helayël-Neto for helpful comments on the paper. This work was partially supported by Fondecyt (Chile) Grant 1130426 and DGIP (UTFSM) internal project USM 111458.

\section{References}

[1] G. Breit and J. A. Wheeler, "Collision of two light quanta," Physical Review, vol. 46, no. 12, pp. 1087-1091, 1934.

[2] S. L. Adler, "Photon splitting and photon dispersion in a strong magnetic field," Annals of Physics, vol. 67, no. 2, pp. 599-647, 1971.

[3] V. Costantini, B. De Tollis, and G. Pistoni, "Nonlinear effects in quantum electrodynamics," Il Nuovo Cimento A, vol. 2, no. 3, pp. 733-787, 1971.

[4] R. Ruffini, G. Vereshchagin, and S.-S. Xue, "Electron-positron pairs in physics and astrophysics: from heavy nuclei to black holes," Physics Reports, vol. 487, no. 1-4, pp. 1-140, 2010.

[5] G. V. Dunne, "The Heisenberg-Euler effective action: 75 years on," International Journal of Modern Physics: Conference Series, vol. 14, pp. 42-56, 2012.

[6] C. Bamber, S. J. Boege, T. Koffas et al., "Studies of nonlinear QED in collisions of $46.6 \mathrm{GeV}$ electrons with intense laser pulses," Physical Review D, vol. 60, no. 9, Article ID 092004, 1999.

[7] D. L. Burke, R. C. Field, G. Horton-Smith et al., "Positron production in multiphoton light-by-light scattering," Physical Review Letters, vol. 79, no. 9, article 1626, 1997.

[8] O. J. Pike, F. MacKenroth, E. G. Hill, and S. J. Rose, "A photonphoton collider in a vacuum hohlraum," Nature Photonics, vol. 8, no. 6, pp. 434-436, 2014.

[9] D. Tommasini, A. Ferrando, H. Michinel, and M. Seco, "Precision tests of QED and non-standard models by searching photon-photon scattering in vacuum with high power lasers," Journal of High Energy Physics, vol. 2009, no. 11, article 043, 2009.

[10] D. Tommasini, A. Ferrando, H. Michinel, and M. Seco, "Detecting photon-photon scattering in vacuum at exawatt lasers," Physical Review A, vol. 77, no. 4, Article ID 042101, 2008.

[11] M. Born and L. Infeld, "Foundations of the new field theory," Proceedings of the Royal Society of London Series A: Containing
Papers of a Mathematical and Physical Character, vol. 144, no. 852, pp. 425-451, 1934.

[12] H. Gies, J. Jaeckel, and A. Ringwald, "Polarized light propagating in a magnetic field as a probe for millicharged fermions," Physical Review Letters, vol. 97, no. 14, Article ID 140402, 2006.

[13] E. Massó and R. Toldrà, "Light spinless particle coupled to photons," Physical Review D, vol. 52, no. 4, pp. 1755-1763, 1995.

[14] P. Gaete and E. I. Guendelman, "Confinement in the presence of external fields and axions," Modern Physics Letters A, vol. 20, no. 5, article 319, 2005.

[15] P. Gaete and E. Spallucci, "Confinement effects from interacting chromo-magnetic and axion fields," Journal of Physics A: Mathematical and General, vol. 39, no. 20, pp. 6021-6029, 2006.

[16] E. S. Fradkin and A. A. Tseytlin, "Non-linear electrodynamics from quantized strings," Physics Letters B, vol. 163, no. 1-4, pp. 123-130, 1985.

[17] E. Bergshoeff, E. Sezgin, C. N. Pope, and P. K. Townsend, "The Born-Infeld action from conformal invariance of the open superstring," Physics Letters B, vol. 188, no. 1, pp. 70-74, 1987.

[18] P. Gaete and J. Helayël-Neto, "Finite field-energy and interparticle potential in logarithmic electrodynamics," The European Physical Journal C, vol. 74, no. 3, article 2816, 2014.

[19] P. Gaete and J. Helayël-Neto, "Remarks on nonlinear electrodynamics," European Physical Journal C, vol. 74, no. 11, article 3182, 2014.

[20] S. H. Hendi, "Asymptotic Reissner-Nordström black holes," Annals of Physics, vol. 333, pp. 282-289, 2013.

[21] Z. Zhao, Q. Pan, S. Chen, and J. Jing, "Notes on holographic superconductor models with the nonlinear electrodynamics," Nuclear Physics B, vol. 871, no. 1, pp. 98-110, 2013.

[22] O. Mišković and R. Olea, "Conserved charges for black holes in Einstein-Gauss-Bonnet gravity coupled to nonlinear electrodynamics in AdS space," Physical Review D, vol. 83, no. 2, Article ID 024011, 2011.

[23] S. H. Mazharimousavi and M. Halilsoy, "Black holes and the classical model of a particle in Einstein non-linear electrodynamics theory," Physics Letters B, vol. 678, no. 4, pp. 407-410, 2009.

[24] G. Amelino-Camelia, "Special treatment," Nature, vol. 418, no. 6893, pp. 34-35, 2002.

[25] T. Jacobson, S. Liberati, and D. Mattingly, “Threshold effects and Planck scale Lorentz violation: combined constraints from high energy astrophysics," Physical Review D, vol. 67, no. 12, Article ID 124011, 2003.

[26] T. J. Konopka and S. A. Major, "Observational limits on quantum geometry effects," New Journal of Physics, vol. 4, pp. 57.1-57.18, 2002.

[27] S. Hossenfelder, "Interpretation of quantum field theories with a minimal length scale," Physical Review D, vol. 73, no. 10, Article ID 105013, 9 pages, 2006.

[28] P. Nicolini, "Noncommutative black holes, the final appeal to quantum gravity: a review," International Journal of Modern Physics A, vol. 24, no. 7, pp. 1229-1308, 2009.

[29] S. Pramanik, S. Ghosh, and P. Pal, "Electrodynamics of a generalized charged particle in doubly special relativity framework," Annals of Physics, vol. 346, pp. 113-128, 2014.

[30] A. F. Ali, S. Das, and E. C. Vagenas, "Discreteness of space from the generalized uncertainty principle," Physics Letters B, vol. 678, no. 5, pp. 497-499, 2009.

[31] G. Amelino-Camelia, "Quantum-spacetime phenomenology," Living Reviews in Relativity, vol. 16, article 5, 2013. 
[32] E. Witten, "Noncommutative geometry and string field theory," Nuclear Physics B, vol. 268, no. 2, pp. 253-294, 1986.

[33] N. Seiberg and E. Witten, "String theory and noncommutative geometry," Journal of High Energy Physics, vol. 1999, article 032, 1999.

[34] M. R. Douglas and N. A. Nekrasov, "Noncommutative field theory," Reviews of Modern Physics, vol. 73, no. 4, pp. 977-1029, 2001.

[35] R. J. Szabo, "Quantum field theory on noncommutative spaces," Physics Reports, vol. 378, no. 4, pp. 207-299, 2003.

[36] J. Gomis, K. Kamimura, and T. Mateos, "Gauge and BRST generators for space-time non-commutative U(1) theory," Journal of High Energy Physics, vol. 2001, no. 3, article 010, 2001.

[37] A. A. Bichl, J. M. Grimstrup, L. Popp, M. Schweda, and R. Wulkenhaar, "Perturbative analysis of the Seiberg-Witten map," International Journal of Modern Physics A, vol. 17, no. 16, pp. 2219-2231, 2002.

[38] A. Smailagic and E. Spallucci, "UV divergence-free QFT on noncommutative plane," Journal of Physics A: Mathematical and General, vol. 36, no. 39, pp. L517-L521, 2003.

[39] A. Smailagic and E. Spallucci, "Feynman path integral on the non-commutative plane," Journal of Physics A: Mathematical and General, vol. 36, no. 33, pp. L467-L471, 2003.

[40] M. Knežević, D. Knežević, and D. Spasojević, "Statistics of equally weighted random paths on a class of self-similar structures," Journal of Physics A: Mathematical and General, vol. 37, no. 1, pp. 1-8, 2004, Erratum in: Journal of Physics A: Mathematical and General, vol. 37, p. 7169, 2004.

[41] E. Spallucci, A. Smailagic, and P. Nicolini, "Trace anomaly on a quantum spacetime manifold," Physical Review D, vol. 73, Article ID 084004, 2006.

[42] R. Banerjee, S. Gangopadhyay, and S. K. Modak, "Voros product, noncommutative Schwarzschild black hole and corrected area law," Physics Letters B, vol. 686, no. 2-3, pp. 181-187, 2010.

[43] A. B. Hammou, M. Lagraa, and M. M. Sheikh-Jabbari, "Coherent state induced star product on $R_{\lambda}^{3}$ and the fuzzy sphere," Physical Review D, vol. 66, no. 2, Article ID 025025, 2002.

[44] L. Modesto, J. W. Moffat, and P. Nicolini, "Black holes in an ultraviolet complete quantum gravity," Physics Letters B, vol. 695, no. 1-4, pp. 397-400, 2011.

[45] P. Nicolini, "Nonlocal and generalizeduncertainty principle black holes," http://arxiv.org/abs/1202.2102.

[46] P. Nicolini, A. Smailagic, and E. Spallucci, "Noncommutative geometry inspired Schwarzschild black hole," Physics Letters B, vol. 632, no. 4, pp. 547-551, 2006.

[47] S. Pramanik, S. Das, and S. Ghosh, "Noncommutative extension of AdS-CFT and holographic superconductors," Physics Letters $B$, vol. 742, pp. 266-273, 2015.

[48] W. Dittrich and M. Reuter, Effective Lagrangians in Quantum Electrodynamics, Springer, Berlin, Germany, 1985.

[49] M. Ostrogradsky, "Memoires sur les equations differentielles relativrs au probleme des isoperimeters," Mémoires de l'Académie Impériale des Sciences de St. Pétersbourg, vol. 4, p. 385, 1850.

[50] P. Gaete and E. Spallucci, "Finite axionic electrodynamics from a new non-commutative approach," Journal of Physics A: Mathematical and Theoretical, vol. 45, no. 6, Article ID 065401, 14 pages, 2012.

[51] P. Gaete, "Some considerations about Podolsky-axionic electrodynamics," International Journal of Modern Physics A, vol. 27, no. 11, Article ID 1250061, 2012.
[52] P. Gaete, "Remarks on gauge-invariant variables and interaction energy in QED," Physical Review D, vol. 59, no. 12, Article ID 127702, 1999.

[53] S. Klarsfeld, "Analytical expressions for the evaluation of vacuum-polarization potentials in muonic atoms," Physics Letters B, vol. 66, no. 1, pp. 86-88, 1977.

[54] A. M. Frolov and D. M. Wardlaw, "Analytical formula for the Uehling potential," The European Physical Journal B, vol. 85, article 348, 2012.

[55] P. Gaete, “On gauge-invariant variables in QED,” Zeitschrift für Physik C Particles and Fields, vol. 76, no. 2, pp. 355-361, 1997.

[56] S. Ferrara and A. Sagnotti, "Massive Born-Infeld and other dual pairs," Journal of High Energy Physics, vol. 2015, no. 4, article 032, 2015.

[57] R. Bufalo, "Born-infeld electrodynamics in very special relativity," Physics Letters B, vol. 746, pp. 251-256, 2015.

[58] H. Kleinert, E. Strobel, and S.-S. Xue, "Fractional effective action at strong electromagnetic fields," Physical Review D, vol. 88, no. 2, Article ID 025049, 2013. 

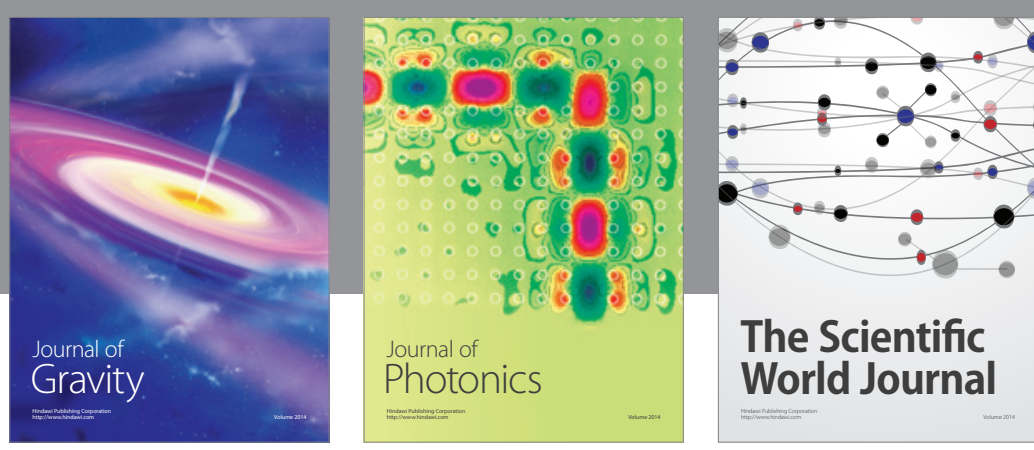

The Scientific World Journal
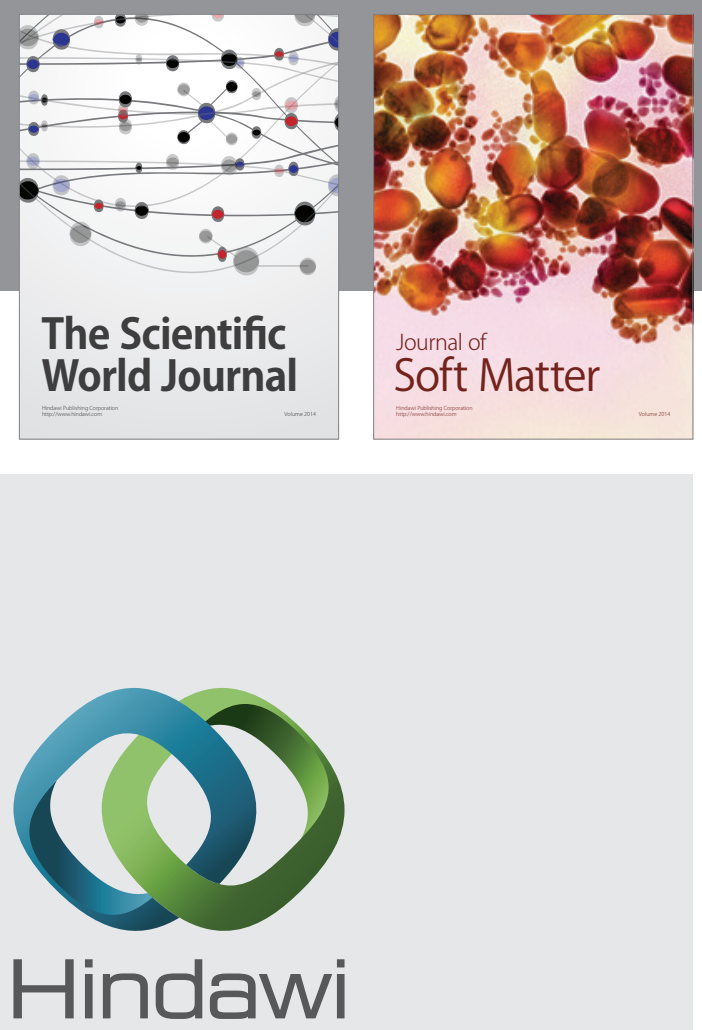

Submit your manuscripts at

http://www.hindawi.com

nternational Journal of

Statistical Mechanics
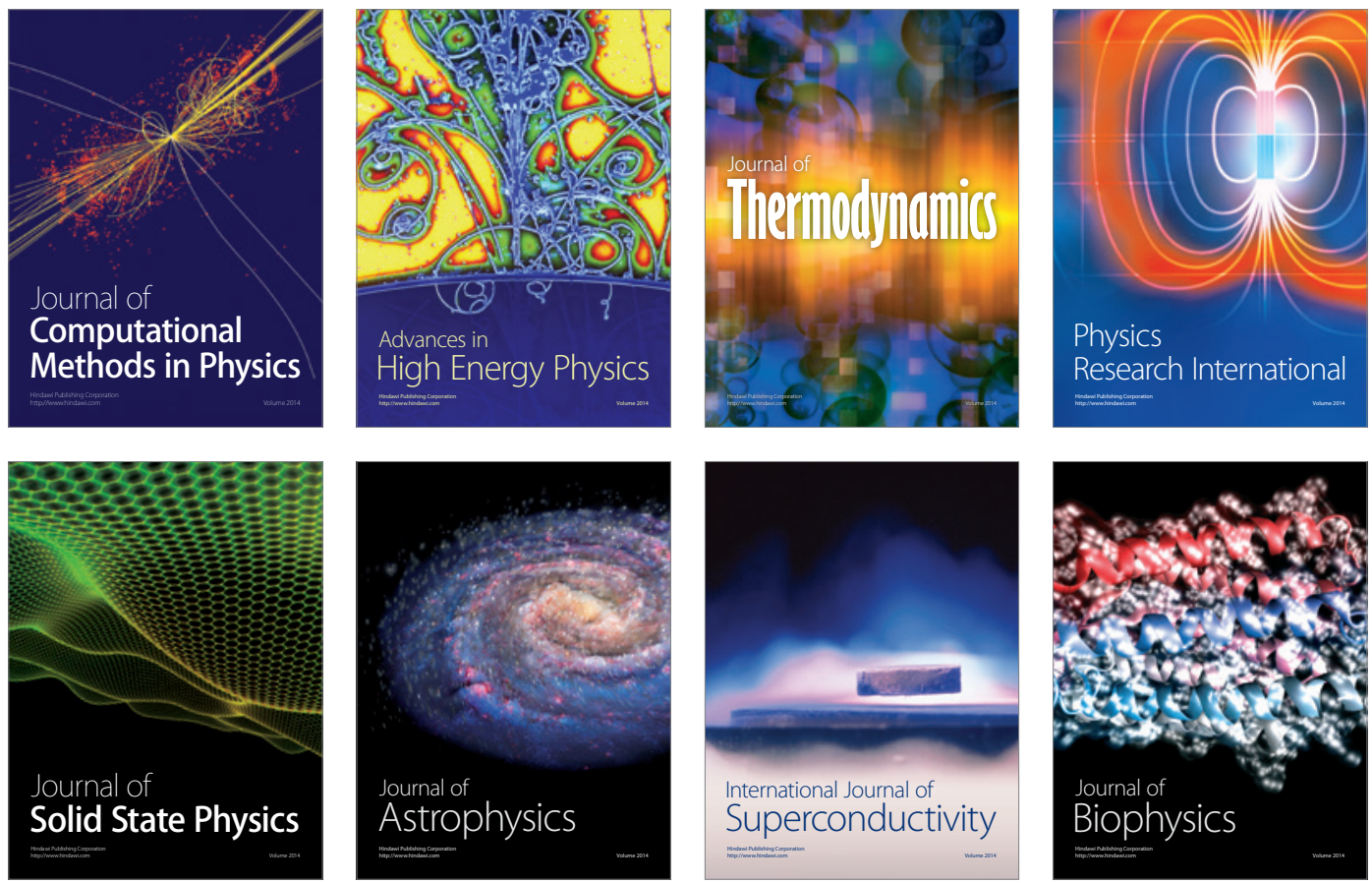
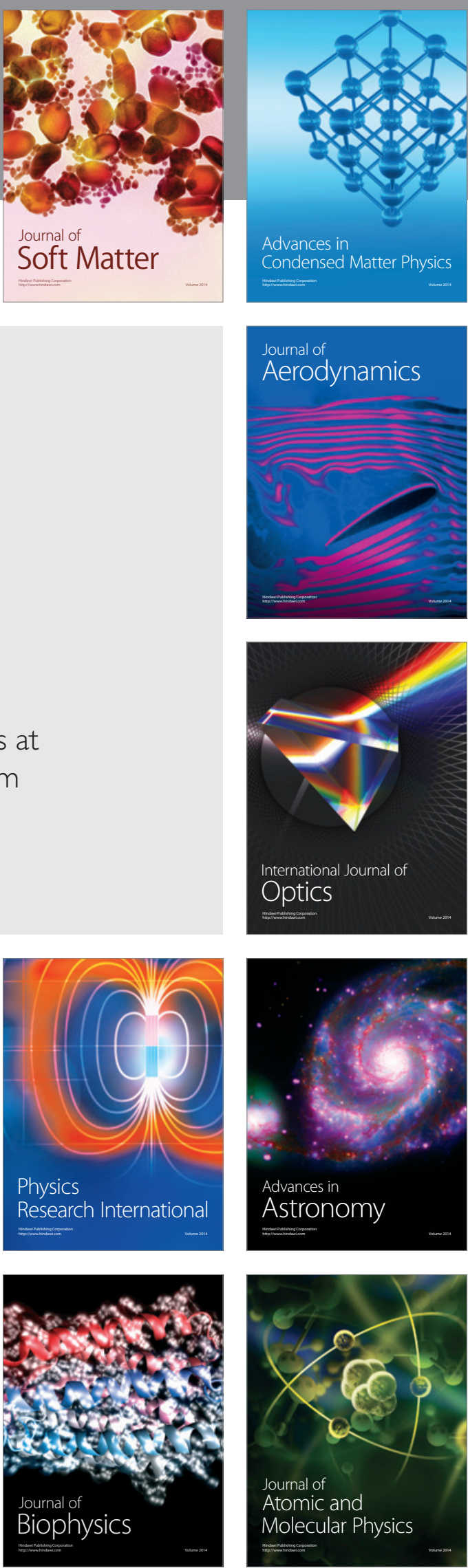